\title{
Blind Deconvolution of Rotational Motion Blurred Image
}

\author{
N. Isoe, R. Maruo, and S. Sugimoto \\ Department of Electrical and Electronic Engineering, Ritsumeikan University \\ Noji-Higashi, Kusatsu City, Shiga 525-8577 Japan \\ Tel: +81-(0)77-561-2673, FAX: +81-(0)77-561-2663 \\ e-mail: sugimoto@se.ritsumei.ac.jp
}

\begin{abstract}
In this paper, we propose a resotration algorithm of rotational motion blurred images using inverse filters. In paticular, to overcome this problem, we focus attention on the two methods. First, we interpolate the pixels near the rotational paths by shift-invariant operation. Second, we reconstruct the input image on the another domain.
\end{abstract}

\section{Introduction}

The rotated motion blur, which is a shift variant blur, often happens in practical situations. Therefore, to restore rotational motion blurred images, it is important to model the degradation process such as relative circular motion between imaging equipments and objects during exposure time. In general, a motion blur is caused by the relative motion between the dijital camera and the captured object. Considering the linear motion blur, the degradation on an image is shift invariant. It shows that it affects the whole parts of an image in the same way. However, rotational motion degradation is shift variant, which shows the degradation affects different parts of an image in differnt ways. Therefore, it is difficult to solve the restoring problem in the shift variant case. Therefore we use the polar coordinate system so that we can regard the shift invariant degradation instead of the shift variant degradation.

First, we introduce the method of shift invariant degradation operation. Second, we explain the shift variant degradation operation. Third, we analyze the motion degradation in the Cartesian system, we estimate the center of rotation using the Hough transform [1]. Then we make a model in the polar coordinate system. Fourth, we estimate the length of blur using cepstrum analysis. Fifth, we construct a model of the line spread function of rotational motion blurs for constant angular velocity cases in the polar coordinate system [2]-[5]. Finally, we introduce the restoration processing using the inverse filter.

\section{Linear Motion Degradation Analysis}

In general, blurred images $g(x, y)$ are described by outputs through PSF(Point Spread Function) from original images $f(x, y)$. In noiseless case, these operations can be expressed as [1] (Fig.1)

$$
\begin{aligned}
g(x, y) & =f(x, y) \otimes h(x, y) \\
& =\int_{-\infty}^{\infty} \int_{-\infty}^{\infty} f(\alpha, \beta) h(x-\alpha, y-\beta) d \alpha d \beta .
\end{aligned}
$$

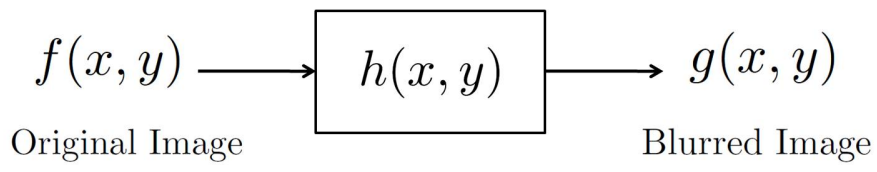

Fig. 1: Model of Degradation process

If velocity $(V)$ of the captured objects is assumed constant during the exposure time $t_{\text {exp }}$ with an angle $\theta$ from the horizon, then the distortion is one dimensional. If $L=V t_{\text {exp }}$, the PSF $h(x, y)$ in spatial domain can be modeled as

$h(x, y)=\left\{\begin{array}{l}\frac{1}{L}, \quad \text { if } 0 \leq|x| \leq L \cos (\theta) ; y=L \sin (\theta) \\ 0, \quad \text { otherwise. }\end{array}\right.$

Suppose that an image $f(x, y)$ affects planar motion, and let $\left(x_{0}(t), y_{0}(t)\right)$ be the time-varying components of motion along $x$-axis and $y$-axis, respectively, and a got image on this process defines $g(x, y)$. The total exposure at any points of the recording medium is obtained by integrating the instantaneous exposure over the time interval while the shutter of camera is open. Then if $t_{\exp }$ is the duration of the exposure, the blurred image in a general form can be defined as [2]

$$
g(x, y)=\frac{1}{t_{\text {exp }}} \int_{0}^{t_{\text {exp }}} f\left(x-x_{0}(t), y-y_{0}(t)\right) d t,
$$

where $x_{0}\left(t_{\text {exp }}\right)=0, y_{0}\left(t_{\text {exp }}\right)=0$. Considering these process, input pixel positions $(x, y)$ move on the linear line during the exposure $t_{\text {exp }}$ in Fig.2. 


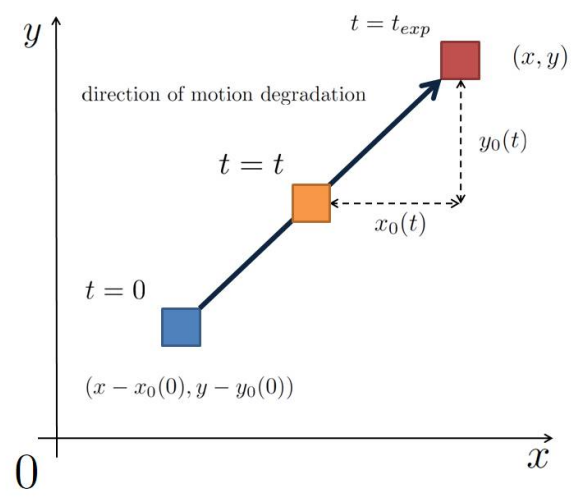

Fig. 2: Concept of linear motion

\section{Rotational Motion Degradation Analysis}

In the Cartesian system, rotational motion blurred image is constructed by the shift variant operation. Fig.3 illustrates the difference between the shift invariant and variant operations in the Cartesian system. Before considering operation, we must consider that this method has the following two problems.

First, for the given angle of rotation, the length of the rotational motion that affects the image pixels will be different for each rotational motion path. Since each rotational motion path has different radius measured from the center of rotation, values of the blur magnitude is influenced by the radius.

Second, each image pixel will be blurred differently depending on its distance to the center of rotation. If the image pixels are further from the center of rotation, the image pixels will be more blurred.

For these reasons, it is impossible to use the PSF as the linear filter and to use (3). Thus, it is necessary to treat the motion degradation on the rotational path as a problem of the linear motion degradation. With that in mind, we consider each input image pixel linearmotioned on the rotational path.


Fig. 3: The difference of shift invariant and shift variant

\subsection{Motion Degradation in Cartesian Coordi- nate System}

Considering the rotational motion blur, the pixels which construct an input image move on the proximity of the rotational path in Fig. 4 where $\left(x_{c}, y_{c}\right)$ means the position of the center of rotation. We introduce the equation (3) in Fig.4 to treat the operation on the rotational path.



Fig. 4: Construction of the pixel position in rotaional motion degradation

Considering to introduce the parameters of radius $r$ and angular displacement $\gamma(0 \leq \gamma \leq \theta)$ in Fig.4, construction of the pixel positions in the Cartesian coordinate space can be developed in the following Fig.4. $\psi(t)$ which means the angular displacement measured from the position of input image pixel at exposure time $t$ can be expressed as

$$
\psi(t)=\omega t
$$

where $\omega$ is the constant angular velocity. In addition, we can write the following references.

$$
\theta=\gamma(t)+\psi(t)
$$

To express the unblurred image by the motion component along the rotational path $(\psi(t))$, we rewrite (5) as

$$
\psi(t)=\theta-\gamma(t)
$$

In general, in the polar coordinate system, any Cartesian $(x, y)$ coordinate points can be expressed as [3]

$$
\begin{aligned}
r & =\sqrt{\left(x-x_{c}\right)^{2}+\left(y-y_{c}\right)^{2}} \\
\theta & =\tan ^{-1}\left(\frac{y-y_{c}}{x-x_{c}}\right) .
\end{aligned}
$$


By using (7) and (8), the radius $r$ and the angular displacement $\gamma(t)$ at exposure time $t\left(0 \leq t \leq t_{\text {exp }}\right)$ can also be expressed as

$$
\begin{aligned}
r & =\sqrt{\left(x-x_{0}(t)-x_{c}\right)^{2}+\left(y-y_{0}(t)-y_{c}\right)^{2}}, \\
\gamma(t) & =\tan ^{-1}\left(\frac{y-y_{0}(t)-y_{c}}{x-x_{0}(t)-x_{c}}\right) .
\end{aligned}
$$

Considering (9) and (10), (3) which means the rotational motion blurred image treated linearly can be expressed as

$$
\begin{aligned}
& g(x, y)=\frac{1}{t_{\exp }} \int_{0}^{t_{e x p}} f\left(r \cos (\gamma(t))+x_{c},\right. \\
& \left.r \sin (\gamma(t))+y_{c}\right) d t .
\end{aligned}
$$

\subsection{Image Description for Polar Coordinate Space}

To consider the motion linearly in the polar coordinate space, the polar coordinate transform process is needed. It can be expressed by using bicubic interpolation of the image pixels in the new domain. Suppose that input image pixels and operated image pixels are on the rotational motion path. If let $r$ maintained and let input image Polar-coordinate-transformed by using bicubic interpolation, it becomes possible to introduce the shift invariant operation to the input image along the $\theta$-axis of the polar coordinate. Therefore, considering Fig.5, Cartesian input image pixels on the rotaional path can be reconstructed linearly in the polar cooridnate space, and can be treated polar pixels blurred linearly. For this reason, the discrete rotaional motion blurred image $z(r, \theta)$ in the space polar coordinate $(r, \theta)$ can be expressed as the summation of unblurred image $u(r, \gamma(t)), t$ from0tot $_{\text {exp }}$ in the space polar coordinate. Namely

$$
z(r, \theta)=\frac{1}{t_{\exp }} \int_{0}^{t_{\text {exp }}} u(r, \gamma(t)) d t
$$

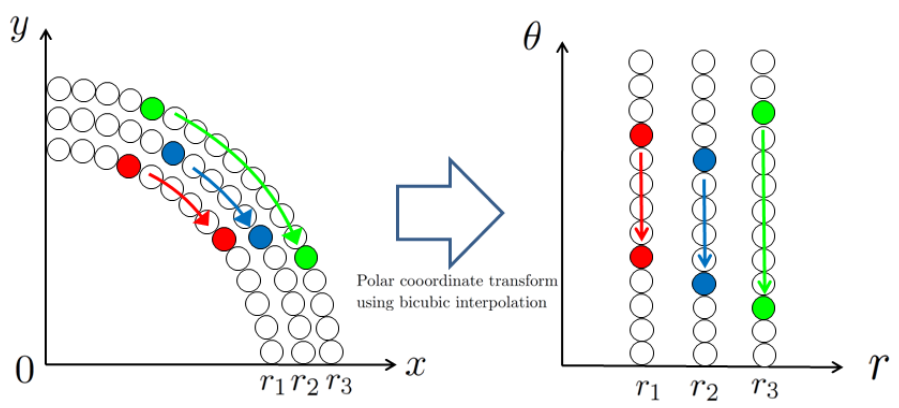

Fig. 5: Polar coordinate tranform

\subsection{Blurring Function Models}

\subsubsection{LSF of the Rotational Mortion Blur}

Although the in-plane rotational motion blurred image is spatially varying in the Cartesian coordinate system, it should be expressed as an output of the shift invariant system to alleviate the restoration. Such consideration can be achieved since the rotational motion blurred image is analogous with the linear motion blur along the rotational path [5]. Here, it is important to express the angular displacement as the arc length. The relation between them can be obtained by using the radius of the rotational path.

By using the variable arc length $\tilde{\omega}=r \omega$ to replace $\omega$, $\tilde{\theta}=r \theta$ to replace $\theta,(4)$ and (6) becomes

$$
\begin{aligned}
\tilde{\psi}(t) & =\tilde{\omega} t \\
\tilde{\psi}(t) & =\tilde{\theta}-\tilde{\gamma}(t)
\end{aligned}
$$

Here, to operate the shift-invariant system in the blurrning paths, it is necessary to express the blurred process by using parameters of the radius and the angle. In the polar coordinate space, by using (12), (13), the blurring process for the constant angular velocity case referring (11) can expressed as [4]

$$
\begin{aligned}
g(r, \tilde{\theta}) & =\frac{1}{t_{\exp }} \int_{0}^{t_{\text {exp }}} f(r, \tilde{\psi}(t)) d t \\
& =\frac{1}{t_{\exp }} \int_{0}^{t_{\exp }} f(r, \tilde{\omega} t) d t
\end{aligned}
$$

From (12), considering the variable of arc length, we have

$$
\frac{d \tilde{\psi}}{d t}=\tilde{\omega} .
$$

Here, it is important to transform $g$ to $g_{r}$, and $f$ to $f_{r}$ for adopting to use shift invariant operation in the polar coordinate space. $g_{r}$ and $f_{r}$ mean an input image and a blurred image on each blurrning path constructed by radius $r$. In addition, they are used in referring to the partial differentiation by radius $r$ to stress the angular displacement of the image pixels.

Thus, by using $g_{r}$ and $f_{r}$, the shift invariant motion blur along the rotational motion path can be expressed in the general form as

$$
g_{r}(\tilde{\theta})=\int_{-\infty}^{\infty} f(\tilde{\psi}) h(\tilde{\theta}-\tilde{\psi}) d \tilde{\psi} .
$$

For each $r$, by using (16), (14) becomes

$$
\begin{aligned}
g_{r}(\tilde{\theta}) & =\frac{1}{t_{\exp }} \int_{\tilde{\theta}-\tilde{\omega} t_{\text {exp }}}^{\tilde{\theta}} f_{r}(\tilde{\psi}) \frac{1}{\tilde{\omega}} d \tilde{\psi} \\
& =\int_{\tilde{\theta}-\tilde{\omega} t_{\text {exp }}}^{\tilde{\theta}} f_{r}(\tilde{\psi}) \frac{1}{\tilde{\omega} t_{\text {exp }}} d \tilde{\psi}
\end{aligned}
$$




$$
\approx \frac{1}{L} \sum_{i=0}^{L-1} f_{r}\left(\tilde{\theta}-i \frac{\tilde{\omega} t_{\text {exp }}}{L-1}\right) .
$$

Either (17) or (19) expresses the one dimensional degradation process. Under the shift invariant degradation process, the line spread function (LSF) of the rotational motion blur along each rotational path can be obtained from (17) and (19). If $l$ is the index of pixels along the rotational motion path, we have

$$
l=\tilde{\psi}
$$

Therefore, the line spread function is given by

$$
h(l)=\left\{\begin{array}{cc}
\frac{1}{\tilde{\omega} t_{\exp }} & \text { for } 0 \leq l \leq \tilde{\omega} t_{\text {exp }} \\
0 & \text { otherwise }
\end{array}\right.
$$

In (20), $L$ is the rectangle function given by

$$
L=\left\lceil\frac{\Phi}{360} 2 \pi r\right\rceil
$$

where $\Phi$ and $\lceil\cdot\rceil$ mean the given the rotation angle and the truncation operation, respectively. By using (23), $\tilde{\omega} t_{\text {exp }}$ in (22) can be expressed as the motion length along the rotational motion path. The length of this rectangle function is $L=\tilde{\omega} t_{\text {exp }}$. As the rotational motion blurred image is spatially invariant in the polar coordinate form, it becomes similar to a linear motion blurred image on the polar coordinate.

\section{Parameter Estimation}

\subsection{Center of Rotation Estimation}

For rotational motion blurred image, it is important to estimate the center of rotation. Degradation image filters using Laplacian filter mask and change this image for binarize image. This binarization image is $g^{\prime}(x, y)$. It is applied Hough transform and extract the center of rotation. $\left(x_{c}, y_{c}\right)$ is the center of rotation. $r$ is radius. $(x, y)$ is point that has pixel value on $g^{\prime}(x, y)$. Then hough transform is defined as follows [1]

$$
\left(x-x_{c}\right)^{2}+\left(y-y_{c}\right)^{2}=r^{2} \text {. }
$$

It transforms $(x, y)$ on the Cartesian coordinates into 3 -D parameters space $\left(x_{c}, y_{c}, r\right)$. So it corresponds to a single plane. If there are many point on Cartesian coordinates, many surfaces can be obtained on 3-D parameters space. Examine the count of the accumulator cells on 3-D parameters space for high pixel concentrations. The highest point is requiring point $\left(\hat{x}_{c}, \hat{y}_{c}, \hat{r}\right)$. So, we determine the center of rotation which is estimated as $\left(\hat{x}_{c}, \hat{y}_{c}\right)$.

\subsection{Motion Length Estimation}

In this paper motion length estimation is done by applying the cepstral analysis. Let $g(x, y), h(x, y)$, and $f(x, y)$ denote the blur image, the PSF, and the original image, respectively. If the blur image is noiseless, then power density spectra of the blurred image is defined as follows.

$$
\left|G\left(\omega_{x}, \omega_{y}\right)\right|^{2}=\left|F\left(\omega_{x}, \omega_{y}\right)\right|^{2}\left|H\left(\omega_{x}, \omega_{y}\right)\right|^{2} .
$$

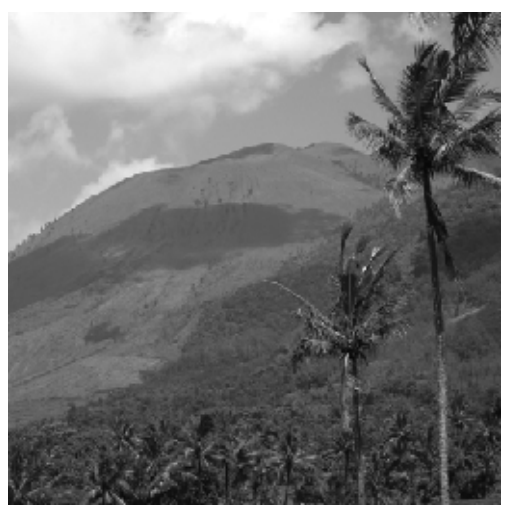

Fig. 6: Original Image



Fig. 7: Fourier Spectrum of Original Image

For the linear motion blur case, cepstrum can be used to estimate the zero patterns in the frequency response from which one can estimate motion direction and motion length. Two-dimensional cepstrum of $g(x, y)$ is used. The power cepstrum is inverse Fourier transform of the logarithm of magnitude of $G\left(\omega_{x}, \omega_{y}\right)$. Thus

$$
\tilde{g}(x, y)=\mathcal{F}^{-1}\left[\log \left\{\left|G\left(\omega_{x}, \omega_{y}\right)\right|\right\}\right]
$$

where $\mathcal{F}^{-1}$ is inverse Fourier transformation. One of important properties of cepstrum is that if two signals are convolved, their cepstrum add. Thus if the noise is neglected

$$
\tilde{g}(x, y)=\tilde{f}(x, y)+\tilde{h}(x, y),
$$




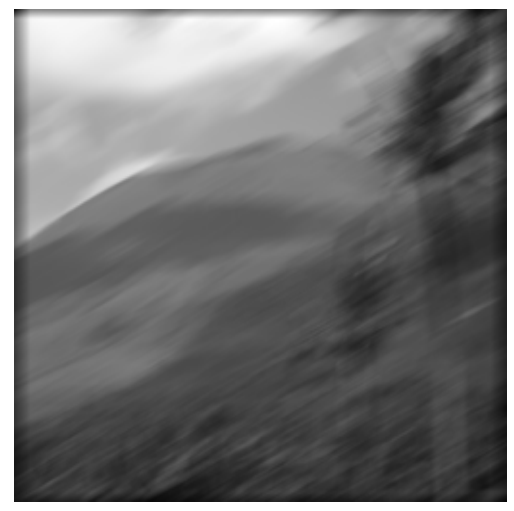

Fig. 8: Motion Blurred Image

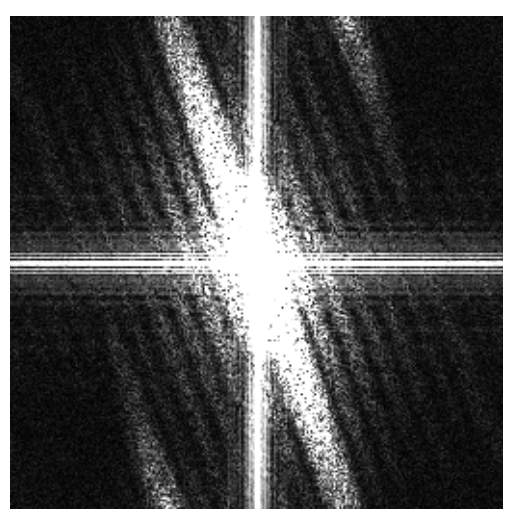

Fig. 9: Fourier Spectrum of Motion Blurred Image

where $\tilde{f}(x, y)$ and $\tilde{h}(x, y)$ denote the cepstra of $f(x, y)$ and $g(x, y)$, respectively.

Fig. 6 and Fig. 8 show an original image and a motion bulrred image, respectively. Also Fig. 7 and Fig. 9 show the Fourier spectra of the images in Fig. 6 and Fig. 8.

When the direction of the linear motion blurred image is known or estimated, we can obtain the onedimensional cepstram of the motion blurred image along the blurred direction. Then from Fig. 10, we can easily find the motion length $\hat{L}$ by operating the cepstram analysis for the motion blurred image in Fig.8.

In Fig. 10, $\tilde{h}$ has a large negative peak at a distance $L$ from the origin. Therefore, the motion length $\hat{L}$ is estimated by calculating the distance of large negative peak from the origin $[6]$.

\section{Image Deconvolution}

The image deconvolution algorithm is developed based on the image degradation model. The one dimensional process in the discrete domain is given by

$$
g_{k_{r}}(n)=h_{0} f_{k_{r}}(n)+h_{1} f_{k_{r}}(n-1)+\cdots
$$



Fig. 10: Cepstral analysis

$$
+h_{L-1} f_{k_{r}}(n-L+1)
$$

where $g_{k_{r}}(n)(n=1,2, \cdots, N)$ are the blurred image pixels in polar coordinate along a rotational motion path with radius $k_{r}, f_{k_{r}}(n)$ are the input image pixels in polar coordinate space, and $N$ is the number of pixels along a rotational motion path, i.e. the concentric circle. In (28), $h_{0}, h_{1}, \cdots, h_{L-1}$ are the coefficients of the line spread function $h(l)$. For the rotational motion blur driven by a non acceleration, $h(l)$ is given by $(23)$. In addition, $L$ coefficients of $h(l)$ have the following relation.

$$
h_{0}=h_{1}=\cdots=h_{L-1}=1
$$

The motion length $L$ is different for the different rotational motion path, and so it is the number of image pixels $N$. (28) shows that a blurred pixel is an accumulation of the line spread function influenced on the corresponding input image pixel and its previous image pixels. The number of image pixels that contibutes to the degradation process is defined as the motion length, i.e. the number of coefficients of $h(l)$.

(28) can be written in a one-dimensional discrete convolution representation as

$$
g_{k_{r}}(n)=\sum_{l=0}^{L-1} h(l) f_{k_{r}}(n-l) \quad n=1,2, \cdots, N .
$$

(30) shows that the blurred image pixel representation for the motion blur driven by a non acceleration.

In noiseless case, if the shift invariant degradation process in (30) is expressed in a matrix form, we get

$$
G_{k_{r}}=H F_{k_{r}}
$$

where $G_{k_{r}}, H$, and $F_{k_{r}}$ is the blurred image pixels, the convolution mask resluting from the line spread function $h(l)$, and the input image pixels, respectively. In (31), $k_{r}$ means the radius of each concentric circle, i.e. $k_{r}=1,2, \cdots, K_{r}$. The size of matrix $H$ operating as the convolution mask for the concentric circle can change as the radius of the concentric circle changes. In addition, 
$G_{k_{r}} \in \mathrm{R}^{M \times 1}, H \in \mathrm{R}^{M \times M}, F_{k_{r}} \in \mathrm{R}^{M \times 1}$ are defined by the following matrices.

$$
\begin{aligned}
& G_{k_{r}}=\left[\begin{array}{c}
g_{k_{r}}(1) \\
g_{k_{r}}(2) \\
\vdots \\
\vdots \\
g_{k_{r}}(M)
\end{array}\right], \quad F_{k_{r}}=\left[\begin{array}{c}
f_{k_{r}}(1) \\
f_{k_{r}}(2) \\
\vdots \\
\vdots \\
f_{k_{r}}(M)
\end{array}\right]
\end{aligned}
$$

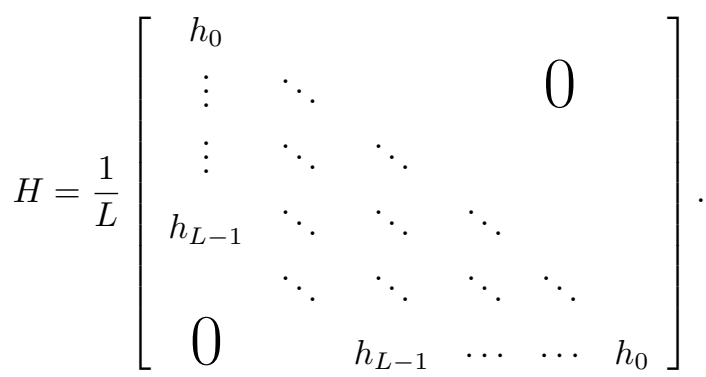

From the aforesaid formula, the restored image can be obtained by employing inverse filtering along the blurring path. Since the degradation process of the rotational motion along blurring path in the polar coordinate system is similar to that of the linear motion blur, the method presented in [?] can be used here. Therefore, the restoration of rotational motion blurred image is realized by applying the folowing equation to each radius $\left(k_{r}=1,2, \ldots K_{r}\right)$,

$$
\hat{F}_{k_{r}}=\hat{H}^{-1} \hat{G}_{k_{r}}
$$



$\hat{G}_{k_{r}}$ is transformed polar coordinate image from degradation image on the center $\left(\hat{x}_{c}, \hat{y}_{c}\right) . \hat{H}^{-1}$ is inverse filter. $\hat{F}_{k_{r}}$ is restored image. However, since the restored image is expressed in the polar coordinate, it is necessary to transform this image for the cartesian coordinate. To overcome this problem, the inverse polar coordinate transform process is applied to the Cartesian restored image to obtain a complete sharp image.

\section{Simulation}

Our experiments are done by using MATLAB 7.4. In our work, we use the Hough transformation for estimating the center of rotation $\left(\hat{x}_{c}, \hat{y}_{c}\right)$. And we use the cepstral analysis for estimating motion length $\hat{L}$.
Table 1 shows the estimation results using our method.

Table 1: Estimation Results

\begin{tabular}{|c|c|c|c|}
\hline $\begin{array}{c}\text { True Value } \\
\text { center of rotation } \\
\left(x_{c}, y_{c}\right)\end{array}$ & $\begin{array}{c}\text { True Value } \\
\text { Motion length } \\
L\end{array}$ & $\begin{array}{c}\text { Estimation } \\
\text { center of rotation } \\
\left(\hat{x}_{c}, \hat{y}_{c}\right)\end{array}$ & $\begin{array}{c}\text { Estimation } \\
\text { Motion length } \\
\hat{L}\end{array}$ \\
\hline$(50,50)$ & 150 & $(54,46)$ & 150 \\
\hline$(100,100)$ & 120 & $(98,96)$ & 123 \\
\hline$(120,60)$ & 130 & $(119,65)$ & 132 \\
\hline$(15,30)$ & 100 & $(21,42)$ & 106 \\
\hline$(128,128)$ & 150 & $(68,114)$ & 29 \\
\hline$(50,50)$ & 30 & $(100,89)$ & 8 \\
\hline
\end{tabular}

In this simulation experiments, we show the results of image deconvolution in Figs. 11 - 14. The original image is shown in Fig. 11. The original image is transformed into the polar coordinated image where bicubic interpolation is applied. Then the rotational blurred image is obtained by transforming into the Cartesian image after summation of $L$-pixels along the $\theta$ direction in the polar coordinate, which is shown in Fig. 12 , where the center of the rotation is assumed as $\left(x_{c}, y_{c}\right)=(50,50)$, and $L=150$. Futher, we obtain the binary image in Fig. 13 by applying the Laplacian operator with 4 neighborhood for edge detection. The Hough transformation is applied for obtaining the arks of the circle so that we can estimate the center of rotation. Table 1 show the estimation results of the center of rotation and the motion blurred length. Finally the restored image using the true value of $\left(x_{c}, y_{c}\right)$ and $L$ is shown in Fig. 14. From the results shown in Table 1, we could not obtain the satesfactory results yet. Futher investigation will be required in the future.

\section{Conclusions}

In this paper, we described the estimation for the center of rortation and the blur length. And we described the restration of rotational motion blurred images using inverse filter. In future work, we will consider more acculate estimation of the center of rotation and more clearly restoration. In this simulation, Because the original image has the same brightness in the four corners, we can get the ideal results of the rotation image in the double precision real operarion by using the PSF of the true values. So we will compare the rotation image for the ideal results and by using the estimated PSF. 


\section{IMAGE \\ PROCESSING}

Fig. 11: Original image

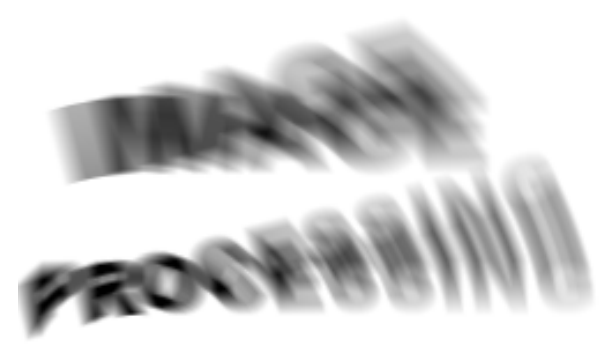

Fig. 12: Rotaional blurred image

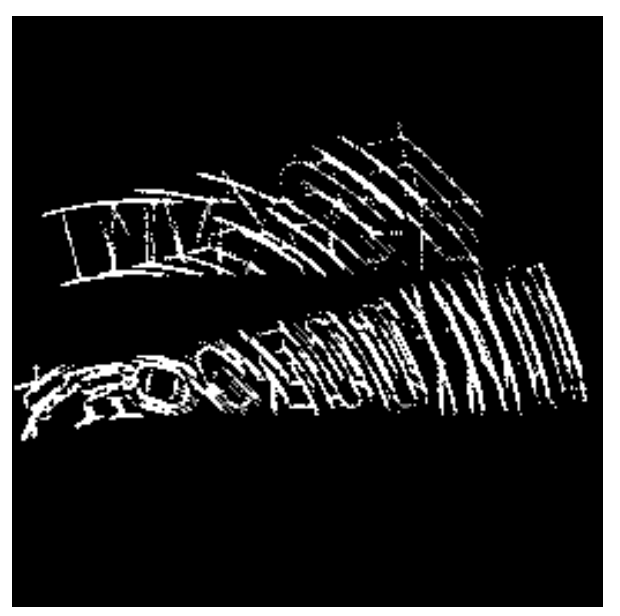

Fig. 13: Rotational image filter Laplacian mask

\section{References}

[1] Rafael C. Gonzalez, Richard E. Woods: Digital Image Processing, 3rd Ed, Peason Prentice Hall,

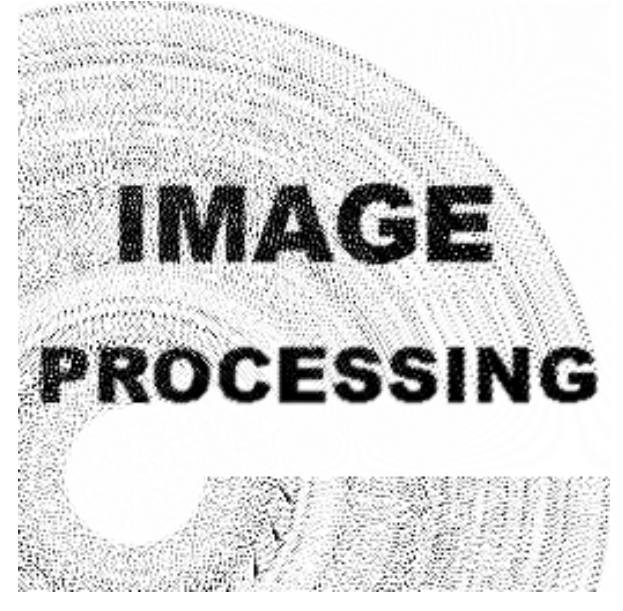

Fig. 14: Restored image

NJ, 2008.

[2] Wikky Fawwaz Al Maki, and S. Sugimoto: Removing Shift Variant Blur without Geometric Coordinate Transform, ISICE Journal of Control, Measurement, and System Integration, Vol.3, No2, pp.67-74, March 2010.

[3] Wikky Fawwaz Al Maki: Blind Deconvolution Algorithms for Rotational Motion Blurred Images, Ph. D Thesis in integrated science and technology Ritsumeikan University, pp.21-30, 62-65, 2009.

[4] Wikky Fawwaz Al Maki, T. Shimahashi, T. Kitagawa, and S. Sugimoto: Space Invariant Blur Estimation and Noiseles Lalman Filter-Based Image Deconvolution, Int. J. of Innovative Computing, Control, and Information, Vol.5, No.1, January, 2009.

[5] Wikky Fawwaz Al Maki, T. Kitagawa, and S. Sugimoto: A Novel Approach to Restore Images Degraded by Rotational Motion Blur, Proc. 52th SCI Conference on Control and Information Systems, pp.177-178, Kyoto, Japan, 2008.

[6] Ioannis M. Rekleities: Optical Flow Recognition from the Power Spectrum of a Signal Blurred Image, IEEE International Conference on Image Proc, 1996, 1996.

[7] W. F. Al Maki, T. Shimahashi, T. Kitagawa, and S. Sugimoto: A New Approach of Blind Image Deconvolution Method without Artifact along the Blurring Paths, Proc. 39th ISCIE International Symposium on Stochastic Systems Theory and Its Application, 2007. 